\title{
Geometrías estructurales aplicadas a la arquitectura de la iglesia de Nuestra Señora de la Candelaria
}

\section{Structural geometries applied to the architecture of the church of Nuestra Señora de la Candelaria}

\author{
Yannette Díaz Umaña ${ }^{1}$ \\ Julio Alfredo Delgado Rojas ${ }^{2}$ \\ Mawency Vergel Ortega ${ }^{3}$ \\ Universidad Francisco de Paula Santander.
}

\section{RESUMEN \\ Este artículo se deriva de la investigación desarrollada entre el programa de matemáticas y el programa de arquitectura de la Universidad Francisco de Paula Santander, cuyo objetivo fue identificar la geometría estructural en la iglesia de}

$1 \quad$ Magister en Gestión Urbana, Arquitecta, docente y directora del Departamento de Arquitectura, diseño y Urbanismo. Filiación: Universidad Francisco de Paula Santander. Correo electrónico: yannettedu@ufps.edu.co Orcid: https://orcid.org/0000-0003-4582-1593

2 Magister en Educación Matemática, Arquitecto, Docente. Filiación: Universidad Francisco de Paula Santander. Correo electrónico: julioalfredo@ufps.edu.co Orcid: https://orcid.org/0000-0001-6944-832X

3 Doctora en Educación. Postdoctora en Imaginarios y representaciones sociales. Docente y Directora del Departamento de Matemáticas y Estadística. Filiación: Universidad Francisco de Paula Santander. Correo electrónico: mawency@ufps.edu.co. Orcid: https://orcid.org/0000-00018285-2968
Nuestra Señora de la Candelaria tomando como punto de partida el análisis del arco catenario, forma ideal para el equilibrio de la estructura. En este proyecto de estudio descriptivo, se analiza las variables del diseño arquitectónico para la arquitectura moderna sagrada, con especial énfasis en las condiciones particulares que hacen de la cubierta una estructura, liviana, resistente y propicia para generar una espacialidad libre de apoyos conforme a los requerimientos espaciales y estéticos de mediados del siglo XX. 


\section{PALABRAS CLAVE}

Arquitectura moderna sagrada, geometría, curvas cónicas, arco catenario.

\begin{abstract}
This article is derived from the research developed between the mathematics program and the architecture program of the Francisco de Paula Santander University, whose objective was to identify the structural geometry in the church of Nuestra Señora de la Candelaria, taking as a starting point the analysis of the catenary arch, ideal shape for the balance of the structure. In this descriptive study project, the variables of the architectural design for modern sacred architecture are analyzed, with special emphasis on the particular conditions that make the roof a structure, light, resistant and conducive to generating a spatiality free of supports according to the spatial and aesthetic requirements of the mid-twentieth century.
\end{abstract}

\section{KEY WORDS}

Modern sacred architecture, geometry, conical curves, catenary arch.

\section{INTRODUCCIÓN}

Este artículo se deriva de la investigación sobre la integración curricular desarrollada entre el programa de matemáticas y el programa de arquitectura de la Universidad Francisco de Paula Santander, cuyo caso de estudio se basó para este caso, en la iglesia de Nuestra Señora de la Candelaria de la ciudad de San José de Cúcuta, ejemplo que brinda la oportunidad de analizar la conceptualización de la geometría vinculada a la estructura y sus características arquitectónicas. El diseño metodológico se plateo teniendo en cuenta el enfoque de la investigación de tipo descriptivo, ya que el interés es cualificar, y describir con un uso natural del lenguaje el objeto de estudio según Sabino (2008), dando una visión general y aproximada de su sentido de habitabilidad para la misión litúrgica.

Es importante señalar, el interés en este tipo de estudios, ya que, la iglesia de Nuestra Señora de la Candelaria de la ciudad de San José de Cúcuta, es una de las primeras en el país con membrana estructural de perfil curvo, modelo representativo, que han sido motivo de estudio en otros contextos, constituyendo una importante contribución a la memoria de la ciudad. Esta construcción de 1952 Vidal (2013), es reconocida a nivel local y nacional debido a su estructura delgada en hormigón de perfil curvo, que la hace distintiva estéticamente y la convierten en una edificación con valor patrimonial. Su sistema constructivo fue usado también para importantes construcciones, según lo comenta la Alcaldía Mayor de Bogotá (2017), como la capilla de los Santos Apóstoles del Gimnasio Moderno en 1.954, iglesia de Fátima en 1.954, iglesia del Liceo Femenino en 1.957, entre algunos de los proyectos patrimoniales de la ciudad de Bogotá, que se encuentran relacionados con los proyectistas de este templo, en la ciudad de Cúcuta. Son ellos, el arquitecto Juvenal Moya y el ingeniero Guillermo González Zuleta, destacados profesionales, cuyas obras se han declarado patrimonio arquitectónico nacional.

En este sentido en Colombia en la década de los 50 , los perfiles urbanos ya mostraban las concepciones del movimiento moderno, incluso en la arquitectura sagrada como se refleja en las obras anteriormente mencionadas. Se debe anotar que el nacimiento de la arquitectura moderna para la arquitectura sagrada, se da en completa sincronización con el Movimiento Litúrgico y todos los fenómenos propios del siglo XIX (Díaz, Delgado, Vergel, 2021), de orden social, económico, cultural y tecnológico, estrechamente ligados a la revolución industrial (Díaz, Delgado, \& Vergel 2021).

Después de todo, el movimiento litúrgico y posterior Concilio, incorporo los avances de 
la modernidad, reafirmado la fe en afinidad con el desarrollo científico-técnico (Díaz y Podetti 2020). A finales del siglo XIX, las innovaciones tecnológicas, el crecimiento de la población, el comercio acelerado, incidieron directamente en la construcción, generando una realización ingenieril notable. Así mismo la producción en serie, nuevas tecnologías y la producción tradicional, desde su fuente como manufactura, se acompañaron en este periodo de eclecticismo histórico. De ahí que “...marca el paso más claro entre el pasado y el presente de la historia de la arquitectura sin el cual es impensable el nacimiento del Movimiento Moderno" (Benévolo,1974, pag.322).

En particular, es debe destacaren la consolidación de la arquitectura moderna, el trabajo de Adolf Loos. Su escrito Ornamento y Delito publicado en 1908 y traducido a varios idiomas conforman una base teórica (Benévolo,1974) nutrida para las siguientes generaciones, en el sentido de buscar las formas sencillas, puras y el rechazo a la ornamentación. En coherencia, se buscaba una arquitectura sencilla y más exequible en términos de dinero. Por otra parte, figuras destacadas en el medio académico y constructivo, que de forma análoga rechazaron el ornato, demuestran la habilidad constructiva que respaldaba de manera magistral esta ideología.

Es el caso de Auguste Perret con la iglesia de Notre Dame du Raincy y Le Corbusier con la iglesia de Notre Dame du Haut en Francia, cuyas formas plásticas en hormigón a la vista, aportaron la expresividad necesaria, para conmover al feligrés, sin el uso del ornamento. Es indudable que esta conquista del hormigón armado, es una victoria no solo francesa sino también alcanzada en otros países europeos y rápidamente américa. En ese sentido, De Fusco (1992) afirma:

"Pero, en realidad, nos encontramos ante un doble compromiso: si la configuración arquitectónica advierte, indudablemente, el uso del hormigón, este a su vez, siendo potencialmente capaz de producir las formas más libres y plásticas, se utiliza por motivos económicos, de más fácil calculo estático y de organización tecnológica más elemental- Renato De Fusco. 1992..." (Pág. 173 y 174)

\section{DISCUSIÓN}

En efecto, el diseño de la iglesia de Nuestra Señora la Candelaria, guarda estas caracterizaciones de sencillez, economía y materialidad constructiva en hormigón. Resulta interesante desde el punto de vista estético, ya que rompe con el esquema tradicional de cruz latina, propio de la iglesia medieval (Delgado, Díaz, \& Vergel, 2.018). Su esquema en planta rectangular, responde a la actualización espacial sugerida por las mociones del Movimiento Litúrgico y a los principios de la arquitectura moderna. Lo que lleva a generalizar, de forma posterior a su construcción, que hacia la década de finales de los 50 las principales ciudades de país ya habían recibido la influencia europea de esta tendencia, no obstante "En Colombia el nuevo ideario formal de la arquitectura entro a operar, por tanto, dentro de lo que era un terreno virgen". (Téllez, 1977, pág. 68).

Se explica de este modo, los efectos de simplificación simbólica de las iglesias en el Siglo $X X$. Se infiere que, la concepción formal de este templo, dispuesto tan solo por dos elementos protagónicos: la membrana con perfil curvo y las superficies acristaladas elaboradas en vidrio coloreados que aportan junto a la piel de hormigón, cualidades de religiosidad artísticas al edificio. Esta composición retorna a los orígenes de la "construcción cristocéntrica de iglesias, al considerar el altar como punto focal de una planta central y una manufactura sencilla con materiales industriales, ajustado a los recursos del lugar, pero que en ultimas lo que busca es 
el efecto fenomenológico en el visitante (Díaz,

Vergel, y Delgado 2020). (imagen 1)
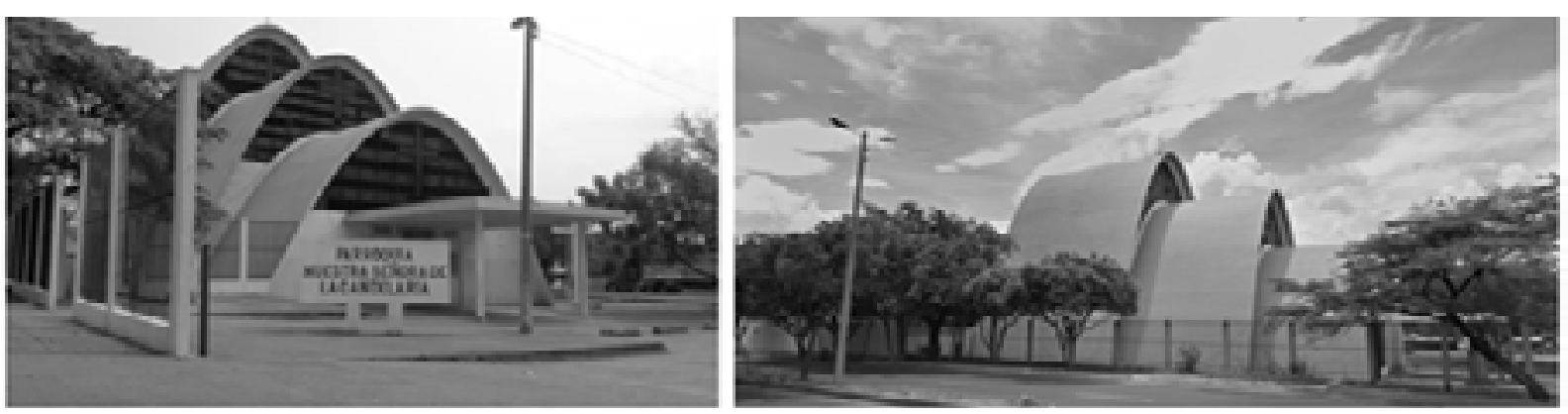

Figura 1. Vista frontal y lateral de la iglesia Nuestra Señora de la Candelaria, San José de Cúcuta,

Colombia. Fuente: Propia.

Con esto, parece confirmar el reto que supuso el diseño arquitectónico del templo, para aportar un lenguaje que de cierta manera sea congruente con el misticismo de los antiguos templos, tal vez habitar, morar y contemplar (Heidegger 1993), como lo consolidaba las cúpulas en los espacios sagrados. Así que la iglesia, no será solo, un objeto simplemente espacial, sino un mediador de la práctica contemplativa, en donde el recurso simbólico y artístico de esta experiencia, facilita descubrir el mensaje y posibilita el encuentro con Dios (Navarro, Castañeda, Peña, y Acero, 2018, pág. 205-210.

En consecuencia, el esquema planímetro, es coherente con las reformas preconciliares que emergen a finales del siglo XVIII, por la necesidad de la modernización de la Iglesia Católica y con el objetivo de renovar la vida litúrgica y la participación más dinámica de la Iglesia (Leon 2019). Por tanto, la conformación de un solo pabellón, en planta libre ${ }^{4}$, concebido sin apoyos internos, permite una total visibilidad al altar de la iglesia de Nuestra Señora la Candelaria, como una franca manera de extender el altar en el recinto, allegando el poder de la celebración a cada fiel. Otro rasgo, es la disposición del coro

$4 \quad$ La planta libre, constituyo un precepto arquitectónico, incluido entre los 5 principios de la arquitectura moderna, propuestos por Le Corbusier, líder del movimiento moderno. Pero también, esta forma rectangular, en la configuración de una sola nave, coincide con las relaciones espaciales, de la zona presbiteral con la audiencia, tal como ocurre en los planteamientos cristocéntricos de las iglesias. en un lugar estratégico, para idear la práctica y participación de la Iglesia en la alabanza, en este caso, se encuentra en los pies de la nave, en un lugar elevado, reubicando su concepción espacial original ${ }^{5}$, en el presbítero, ya que eran únicamente los del clero, que cantaban los oficios divinos. (imagen 2)

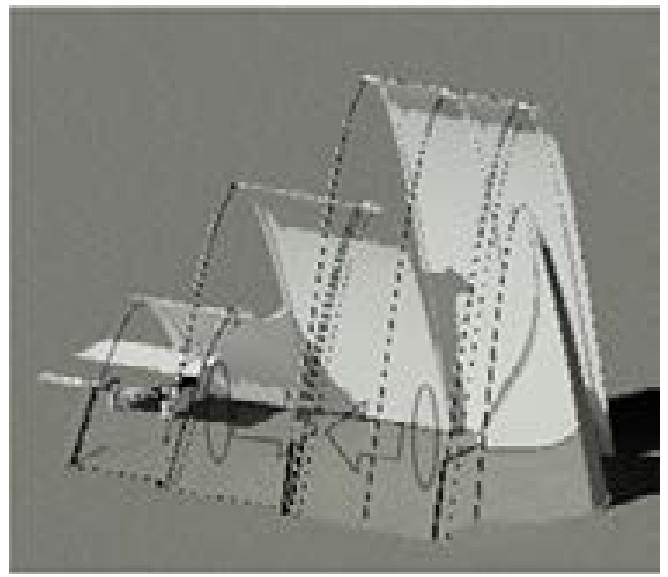

Figura 2. Modelado arquitectónico de la iglesia Nuestra Señora de la Candelaria. Descubre la relación de las cubiertas con perfil curvo con la planta libre, revelando su unidad interna.

Fuente: Propia.

En cuanto a las tres membranas como cubierta del templo de Nuestra Señora la Candelaria, que constituyen la piel arquitectónica, integradas en un solo gesto plástico, sobre su planta rectangular. La organización en el interior es

$5 \quad$ Se refiere a la ubicación del coro en los antiguos templos. 
sencilla, simétrica y orientada a una sola nave, por lo que la expresividad de su envoltura le confiere singularidad. Resuelve precisamente esta envoltura con tres estructuras laminares de perfil curvo de elevación progresiva, de tal forma que la medida lograda para la estructura de menor dimensión es de 18 metros de luz y 10 metros de altura (Figura 5, ecuación 3), le continua 20 metros de luz y 15 metros de altura para la membrana intermedia (Figura 5 ecuación 4), y la más alta que llega a los 22 metros de luz y alcanza los 20 metros de altura (Figura 5 ecuación 5).
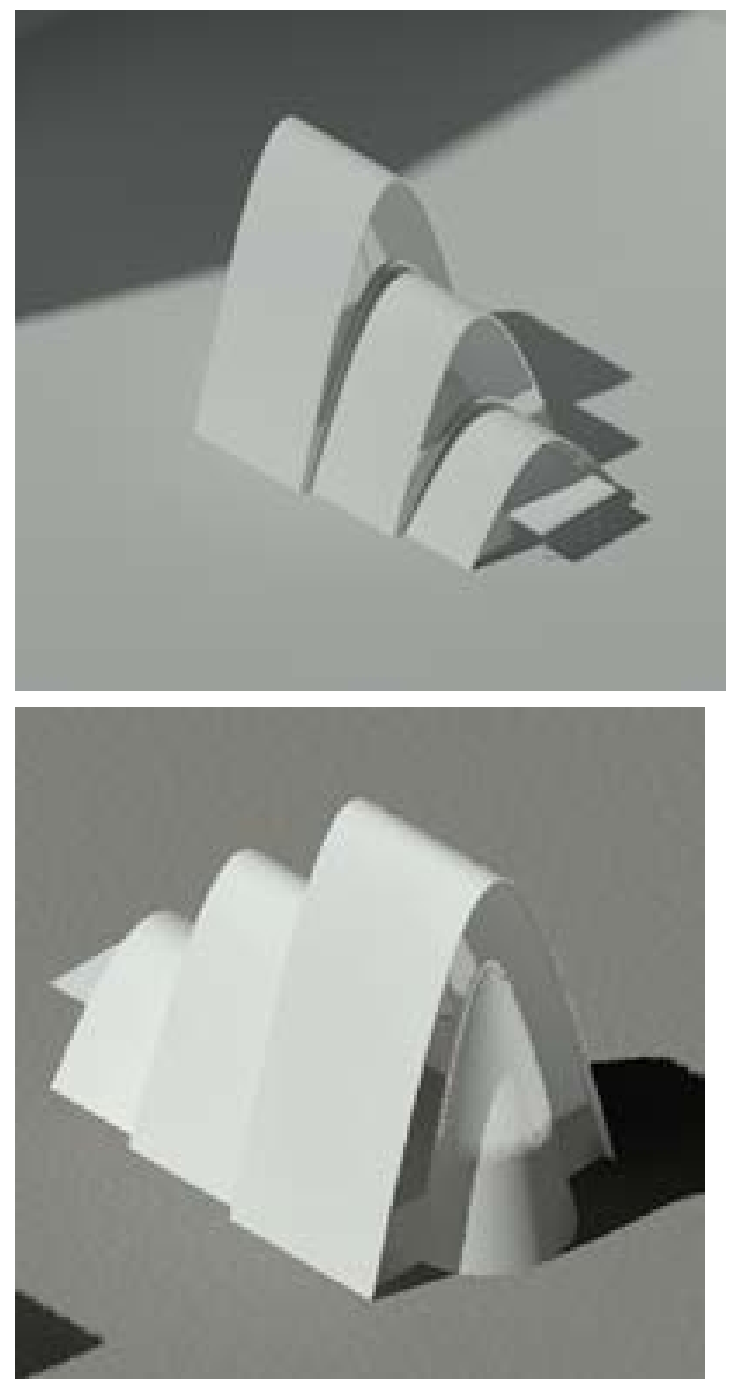

Figura 3. Modelado arquitectónico de la iglesia Nuestra Señora de la Candelaria. Vistas de las tres estructuras laminares de perfil curvo de elevación progresiva. Fuente: Propia.
Este envolvente, tienen un espesor de $10 \mathrm{~cm}$, es auto portante, resistente al paso del tiempo y con especiales propiedades acústicas. El Ingeniero Gonzales, el calculista propuso, mediante un entramado de viguetillas de hormigón armado de $10 \mathrm{~cm}$ de sección, cada una con cuatro varillas redondas de acero de 1/4" (Galindo, Salazar, y Henao, 2018, pág. 4). Adicionalmente se encuentran otros elementos estructurales de $84 \mathrm{~cm}$ y $28 \mathrm{~cm}$, que le confieren también la estabilidad, al perfil. De tal modo que, entre ellas, se acomodan ladrillos de arcilla de $23 \mathrm{~cm}$ de ancho y $34 \mathrm{~cm}$ de largo, con las características propias de producción artesanal y cuya porosidad gana poco peso. En general lo que se observa en esta estructura es que la tracción que padece cada punto del arco se reparte entre una componente vertical y una componente de presión que se transmite a través del propio arco hacia los cimientos, sin que se creen esfuerzos horizontales, salvo en el extremo llegando ya a los cimientos. Es esta propiedad la que hace que los arcos catenarios no necesiten apoyos laterales para sustentarse.

De todas formas, el enlucido de mortero favorable para la impermeabilización, aplicado en la cara externa de la membrana curvada, la hace aparentar ser una estructura totalmente en hormigón, y de esta manera evidenciar la coherencia con los ideales modernos. De acuerdo a esta caracterización, el modernismo en localidades como San Jose de Cúcuta, resulta ser ecléctico también a su modo, aportando en una sola obra, varios conceptos y una variedad de materiales vernáculos, manufacturados de forma artesanal, y los industrializados, conformes a los referentes europeos. Lo cierto es que, este sistema constructivo propuesto por Gonzales Zuleta, resultaría para aquella época innovador por su respuesta netamente geométrica.

En referencia a esto, la composición de las bóvedas ligeramente desplazadas para el 
paso de iluminación a la nave, dan espacio a las superficies acristaladas que actúan como tímpanos elaborado con vitrales. Este distintivo revela que, aunque la obra haya pretendido romper con los esquemas estilísticos de la arquitectura sagrada medieval, se adapta la tradición del uso del vitral (Vallespín, Hernández, y Cervero, 2019), más que elemento decorativo, es un elemento cargado de simbolismo y religiosidad (Díaz, Vergel, y Delgado 2020), tal como era su objetivo en los templos góticos. En realidad, esta práctica no fue extraña en Colombia, en ocasiones los arquitectos tornaban al uso de alusiones históricas en sus diseños, como táctica respetable para engalanar la evidente novedad de sus conceptos espaciales (Téllez 1977).

De acuerdo a lo anterior, el caso del estudio de la iglesia de Nuestra Señora de la Candelaria, en particular, la solución estructural en aquella época de los años 50 la hace un ejemplo arquitectónico único. Como se sabe, la estructura ha sido un componente vital de la arquitectura, en este caso una delgada lamina curva de hormigón permite consolidar un espacio interno óptimo para la celebración litúrgica. Su estudio se concentra en su geometría, cuyas curvas logradas por el entramado de armaduras de acero y en este caso, también piezas de material cerámico, tal como lo han diseñado algunos arquitectos a nivel mundial, bien sea Antonio Gaudí o Félix Candela, quienes propusieron exitosamente estas curvas estructurales.

Es de advertir que se puede observar en un cono de revolución, la línea recta llamada generatriz, cuyo movimiento es fijo en un punto alrededor de un eje circular llamado directriz, que, en los cortes, se obtienen diferentes tipos de curva, de origen cónico, como la elipse, la hipérbola y la parábola (Figura 4). De acuerdo a estas características geométricas, es importante resaltar que existe otro tipo de figura, la curva catenaria. En matemáticas y arquitectura se recurre a la palabra catenaria para distinguir la curva que sigue la forma que adquiere una cadena o cuerda de densidad uniforme y perfectamente flexible sujeta por sus dos extremos y que se encuentra sometida únicamente a las fuerzas de la gravedad (de Zárraga, 2003). Esta forma es usada en la arquitectura e ingeniería muy resistente a la tracción y compresión en respuesta a las fuerzas generadas por la gravedad.
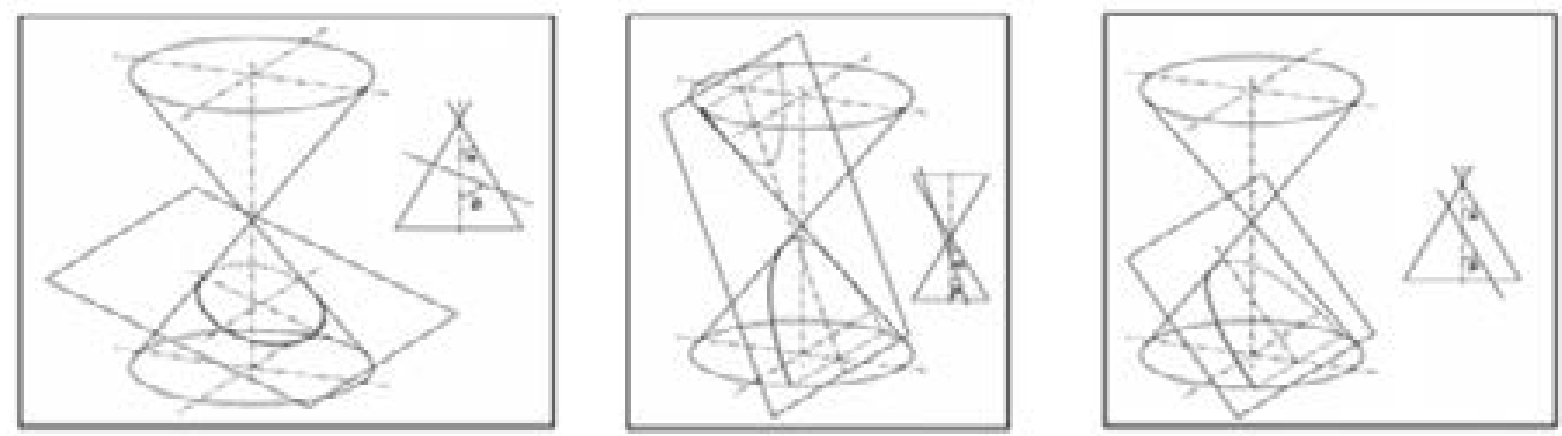

Figura 4. Tipos de curva de origen cónico: elipse, hipérbola y parábola.

Fuente: elipse, hipérbola, y parábola de Flores, Nicolas y Oliva, Gerardo (2016). 
En la imagen 4 se puede observar como el trazado de la parábola se asemeja mucho al trazado de la catenaria, no obstante, estas curvas son diferentes, pues mientras la parábola está definida por una ecuación cuadrática, en la expresión de la catenaria se incluyen funciones hiperbólicas según lo comentado por de Zárraga (2003). Esta autora también menciona el matemático alemán Joachin Jungius en 1669, que fue capaz de demostrar que una cadena colgante no adoptaba una forma de parábola. Para comprender las diferencias entre la catenaria y una parábola, se puede decir por un lado que, en el caso de la catenaria, el valor de la tangente tiende a la verticalidad, mientras que en la parábola este valor tiene a una constante (Imagen 5). Esto condiciona que, en la catenaria, para valores infinitos de la $\mathrm{y}$, la $\mathrm{x}$ tiende a valores limitados, mientras que en la parábola para los valores infinitos de la y se obtienen valores infinitos de la $x$.
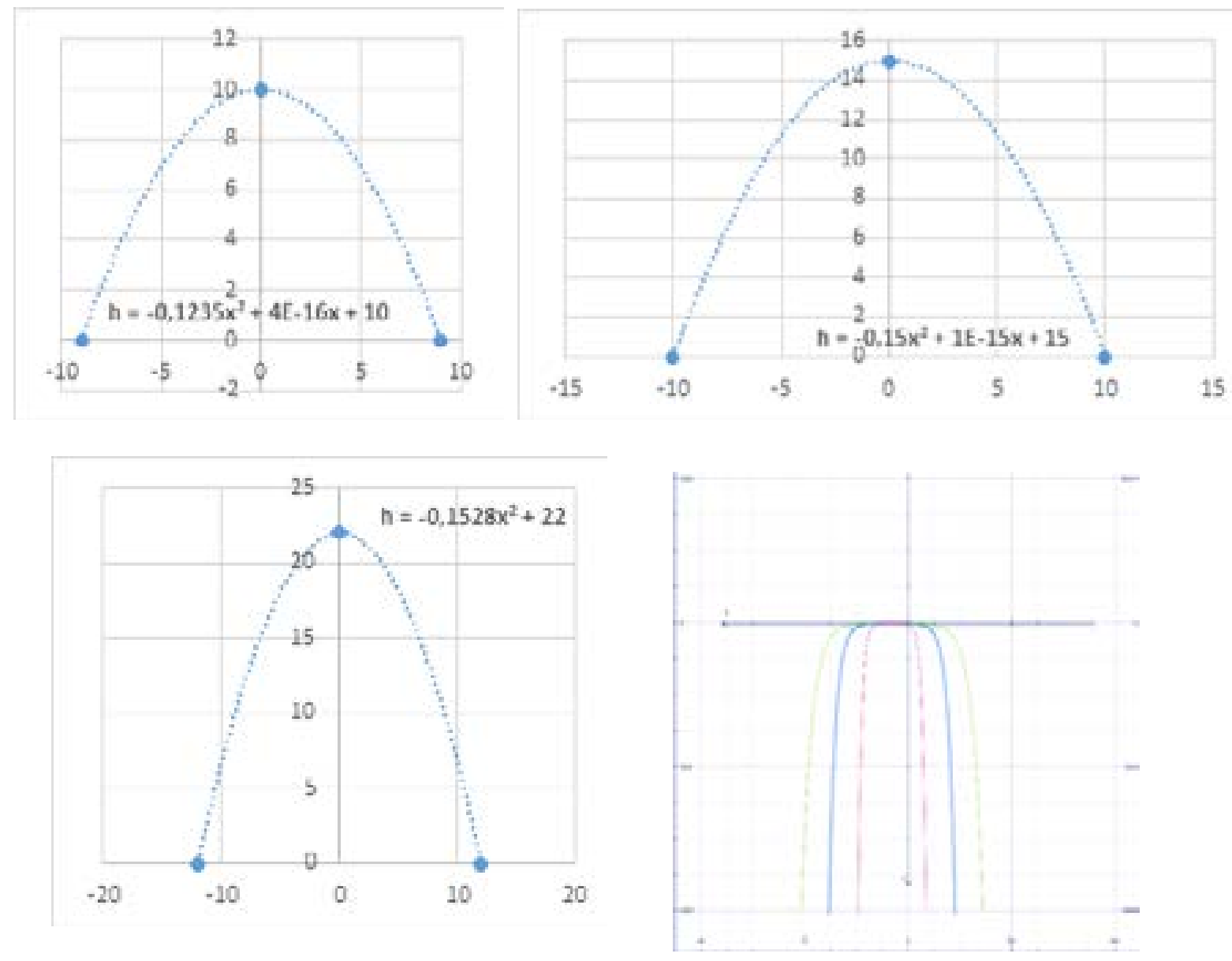

Imagen 5. Comparación de las curvas catenaria y parábola. Fuente: Autores

En consecuencia, la fórmula matemática de estas dos curvas tienen similitudes, pero los últimos términos de esta expresión se fórmula $x$ elevadas a potencias mayores, en el caso de la catenaria. De esta manera se pueden distinguir su fórmula: 
Ecuación de la catenaria:

$$
y=\alpha \operatorname{Cosh}\left(\left(x-x_{0}\right) / \alpha\right)+y_{0}
$$

Asociada a la ecuación de la parábola:

$$
\begin{aligned}
& y=a \cdot x^{2}+b \cdot x+c \\
& h=-0,1235 x^{2}+4 E-16 x+10 \\
& h=-0,15 x^{2}+1 E-15 x+15 \\
& h=-0,1528 x^{2}+22
\end{aligned}
$$

Aplicando este principio en la estructura del caso de estudio, el arco que reproduce exactamente la morfología de la Iglesia de Nuestra señora de la Candelaria es una curva catenaria invertida, denominada arco catenario. Es decir, matemáticamente las características de la catenaria se conservan al invertir su gráfica. De modo que el arco catenario es la forma ideal para sobrellevar su propio peso, como se presenta en este caso. Cuando está construido de elementos individuales, como el caso de las más de 6000 piezas de arcilla, cuya direccionalidad es perpendiculares a la curva del arco, no existen fuerzas de cizalla significativas en las uniones y la fuerza al apoyo se transmite a lo largo de la línea del arco. Posee la ventaja que cuando mayor es la altura, más pequeño es el empuje horizontal en los puntos de arranque, con lo que se pueden obtener grandes alturas con mínimos empujes laterales, de ahí la estructura más alta posee mayor resistencia en sus laterales. La transmisión de esfuerzos y presiones hacia los cimientos es la propiedad que hace que los arcos catenarios de esta iglesia no necesiten apoyos laterales para sustentarse.

\section{CONCLUSIÓN}

Tanto el Movimiento Litúrgico y posterior Concilio, incorpora los avances de la modernidad, reafirmado la fe en afinidad con el desarrollo científico y tecnológico. Por otra parte, el movimiento arquitectónico moderno, también formula el propósito de renovación, ya que, se encamina a la austeridad y la simplificación constructiva haciendo uso de la geometría. Con este contexto, se presenta la iglesia de Nuestra Señora de la Candelaria, como un ejemplo tipológico en la ciudad de Cúcuta. No solo por su innovación tecnología al aplicar el arco catenario en el perfil de hormigón, sino por la conceptualización de las iniciativas del Movimiento Litúrgico en la espacialidad del recinto sagrado, teniendo en cuenta su fecha de inauguración, como sello de su sentido vanguardista. De donde resulta, una planta absolutamente libre de apoyos para facilitar la integración de la zona presbiteral con la audiencia, por tanto, extender el poder del altar a cada uno de los fieles. Luego, facilita la experiencia mística, despreciando la concepción de mero objeto espacial, para vincularse como mediador del hábito contemplativo incluso también con el uso de formas geométricas relacionadas con el origen cónico.

De este modo, la geometría estructural se consigue con una técnica constructiva propia de la modernidad, al invertir la curva catenaria, conservando las características matemáticas. El denominado arco catenario, como se le conoce, es la forma ideal para un cuerpo que se soporta a sí mismo. Cuando está construido de elementos individuales cuyas superficies son perpendiculares a la curva del arco, cuyos esfuerzos y cargas son directamente llevados a los cimientos. Su estabilidad no disminuye al crecer en altura, por el contrario, es una forma geométrica muy estable sin que se requieran apoyos complementarios. 


\section{REFERENCIAS BIBLIOGRÁFICAS}

Alcaldía Mayor de Bogotá. (2017). Arquitectura sublime. El patrimonio religioso de Bogotá. Bogotá: Buenos y Creativos S.A.S.

Benévolo, Leonardo. (1974) Historia de la Arquitectura moderna. Barcelona: Editorial Gustavo Gili.

Díaz, Bárbara y Podetti, Ramiro. Protestantismo y Concilio Vaticano II: una original tesis de Alberto Methol Ferré. Franciscanum173, Vol. 62 (2020): 1-18

Delgado, A., Díaz, Y. \& Vergel, M. (2.018). El paisaje Arquitectónico y sonoro del campanario de la Catedral de San José de Cúcuta. Revista Logos Ciencia \& Tecnología.vol.11 $\mathrm{N} \mathrm{1}$, enero - marzo 2.019 P52-60v

Delgado, Alfredo; Díaz, Yannette y Vergel, Mawency (2019). El Paisaje Arquitectónico y sonoro del Campanario de la Catedral de San José de Cúcuta. Revista Logos Ciencia \& Tecnología, vol. 11, no. 1, (2019):52-60. Consulta en diciembre 10 2019: file:///C:/Users/ufps/ Downloads/801-Texto\%20del\%20art\% C3\%ADculo-4700-1-10-20190126.pdf

Díaz Umaña, Y.., Delgado Rojas, J. A.., \& Vergel Ortega, M.. (2021). Representaciones socioespaciales, en la memoria histórica de la catedral y su entorno urbano en San José de Cúcuta. Revista Boletín Redipe, 10(4), 376-387. https://doi. org/10.36260/rbr.v10i4.1277

Díaz Umaña, Y.., Delgado Rojas, J. A.., \& Vergel Ortega, M.. (2021). Representaciones socioespaciales, en la memoria histórica de la catedral y su entorno urbano en San José de Cúcuta. Revista
Díaz, Y., Vergel, M. y Delgado, R. (2021). Entre las torres de San José. Aproximaciones al arte, arquitectura y geometría de la catedral. Bogotá: Ecoe ediciones.

Estivill Daniel. (2019) Apuntes sobre arquitectura sacra., Cuestiones Teológicas Vol. 41, No. 95, Medellín-Colombia. Enerojunio 2014, 41-74, $58 . \quad$ Consulta en noviembre 3 2019: https://revistas.upb. edu.co/index.php/cuestiones/article/ view/2250/2013

Galindo Díaz, Jorge, Salazar Marulanda Carolina, Henao Laura. (2018). Cubiertas laminares en cerámica armada: los aportes del ingeniero Guillermo González Zuleta (Colombia, 1947-1962). (Informes de la Construcción, 70,551, (2018): 1-12. Consulta en noviembre 26 2019: https://doi.org/10.3989/ic.60713

Heidegger Martin. (1993). Construir, habitar y pensar. Santiago de chile: editorial Universitaria.

Renato De Fusco. (1992). Historia de la Arquitectura contemporánea. Madrid: Celeste Ediciones.

León, Leticia (2019). Espacios Sagrados después del Concilio Vaticano II: Templos católicos en Morelia. Actas del congreso Internacional de arquitectura Religiosa contemporánea 4. 2015, 102107. Consulta en noviembre 10 2019: https://dialnet.unirioja.es/descarga/ articulo/5849038.pdf

Loos Adolf. "Ornamento y delito". (2019). paperback $n^{0} 7$. ISSN 1885-8007. 1908. Consulta en diciembre 3 2019: http:// www. paperback.es/articulos/loos/ ornamento.pdf 
Martinez, Ezequiel (s.f.). Curva catenaria y parábola. Parábola. Sus graficas con vértice en común. Visto en: https://www. geogebra.org/m/cwub2bRZ

Navarro Sánchez, Rosana; Castañeda, Fredy Humberto; Peña Esquivel, William Augusto y Acero Montañez, José Ricardo. (2018). «El acontecer del misterio en el silencio, la contemplación y representación». Franciscanum170, Vol. Ix (2018): 183-214

Sabino, Carlos (2008). El proceso de Investigación. Caracas: 17 Editorial Panapo.

Téllez, German (1977). Crítica \& Imagen. Bogotá: ESCALA Fondo Editorial.

Vallespín, Aurelio; Hernández, Luis y Cervero Noelia (2019). El espacio Gótico según Hans Jantzen y la evolución de las vidrieras. Expresión gráfica arquitectónica Universidad Politécnica de Valencia.(2015):238- 247. Consulta en noviembre 26 2019: https://doi. org/10.4995/ega.2015.3671

Vidal Julio César (2013). Para la verdad. Diócesis de san José de Cúcuta. Cúcuta,

Flores, Nicolas y Oliva, Gerardo (2016). Arquitectura sustentable con definición de patrones de comportamiento estructurales en materiales y sistemas de materiales actuales. Tlamati Sabiduria, Volumen 7 Número Especial 2 visto en: http://tlamati.uagro.mx/t7e2/60.pdf

de Zárraga Mata, Susana (2003). LA CATENARIA EN. ARQUITECTURA .EI cálculo de estructuras en la obra de Gaudí. Ingenieria civil 2003; 129: 12133. 